\title{
Hydrazine-Cadmium Tellurite Hybrid Microcrystals: An Efficient Precursor to Porous Cadmium Telluride and Tellurium Architectures Through Its Thermal Decomposition
}

\author{
Hong-Bin Yao, Xiao-Bo Li, Si-Yuan Ai, and Shu-Hong Yu (ه) \\ Division of Nanomaterials and Chemistry, Hefei National Laboratory for Physical Sciences at Microscale, Department of Chemistry, \\ University of Science and Technology of China, Hefei 230026, China \\ Received: 27 September 2009 / Revised: 1 December 2009 / Accepted: 2 December 2009 \\ C The Author(s) 2010. This article is published with open access at Springerlink.com
}

\begin{abstract}
Well-defined platelet-like hydrazine-cadmium tellurite hybrid microcrystals have been synthesized by a solvothermal reaction of cadmium chloride, sodium tellurite, and hydrazine hydrate in a mixed solvent containing $n$-propylamine and deionized water. The formula of the hybrid platelet-like microcrystals has been proposed based on a combination of powder X-ray diffraction pattern (PXRD), elemental analysis, thermogravimetic analysis (TGA), and X-ray photoelectron spectroscopy (XPS). Controlled thermal decomposition of this hybrid precursor can lead to the formation of porous platelet-like microarchitectures. Pure porous cadmium telluride architectures were obtained by using hydrochloric acid to dissolve $\mathrm{CdTeO}_{3}$ remaining in the sample after thermal decomposition at $450^{\circ} \mathrm{C}$. In addition, unique nanoporous tellurium architectures were obtained by using hydrochloric acid to dissolve the amorphous $\mathrm{Cd}\left(\mathrm{N}_{2} \mathrm{H}_{4}\right) \mathrm{TeO}_{3}$ formed after thermal decomposition at $300{ }^{\circ} \mathrm{C}$, followed by an in situ topotactic reaction between the residual three-dimensional (3-D) skeleton of cadmium telluride nanocrystallites and $\mathrm{TeO}_{3}^{2-}$. Brunauer-Emmett-Teller (BET) analysis and a study of the optical properties of these porous cadmium telluride and tellurium materials have also been carried out.
\end{abstract}

\section{KEYWORDS}

Thermal decomposition, hydrazine, hybrid, porous architecture, tellurium

\section{Introduction}

As novel materials combining two or more components, hybrid materials have been extensively studied in fundamental research and applied in industry in recent years [1]. For example, a variety of organoamine-metal chalcogenide organic-inorganic hybrid materials have been synthesized and shown to have novel optical, electrical, and thermal expansion properties [2-12]. In particular, various hydrazine- based hybrid materials can be synthesized by using hydrazine as both solvent and reagent because of its high solubility and reducing capability toward chalcogenide elements. For example, Mitzi and his co-workers have reported a series of hydrazinebased hybrid materials including $\left(\mathrm{N}_{2} \mathrm{H}_{5}\right)_{4} \mathrm{Sn}_{2} \mathrm{~S}_{6}$ [13], $\left(\mathrm{N}_{2} \mathrm{H}_{5}\right)_{4} \mathrm{Ge}_{2} \mathrm{Se}_{6}$ and $\left(\mathrm{N}_{2} \mathrm{H}_{4}\right)_{3}\left(\mathrm{~N}_{2} \mathrm{H}_{5}\right)_{4} \mathrm{Sn}_{2} \mathrm{Se}_{6}$ [14], $\alpha-$ and $\beta$ - $\left(\mathrm{N}_{2} \mathrm{H}_{4}\right)_{2} \mathrm{ZnTe}$ [15], and $\left(\mathrm{N}_{2} \mathrm{H}_{4}\right)\left(\mathrm{N}_{2} \mathrm{H}_{5}\right) \mathrm{Cu}_{7} \mathrm{~S}_{4}$ [16]. Yuan et al. have also systematically investigated a series of structures of hydrazine-based metal chalcogenide

$\overline{\text { Address correspondence to shyu@ }}$ ustc.edu.cn 
hybrids spanning from zero-dimensional (0-D) discrete molecules, to 1-D chains, 2-D layers, and 3-D networks [17]. In addition, Li's group have reported a layered monohydrazine compound $\mathrm{ZnTe}\left(\mathrm{N}_{2} \mathrm{H}_{4}\right)$ [4]. Recently, a new hydrazine-metal chalcogenide hybrid framework material, $\mathrm{Mn}_{2} \mathrm{SnS}_{4}\left(\mathrm{~N}_{2} \mathrm{H}_{4}\right)_{2}$, in which the hydrazine acts as an integral part of the framework as opposed to being located in the pores, has been reported by Kanatzidis et al. [18].

Hydrazine-based hybrid materials can be used as efficient precursors for the fabrication of functional inorganic substrates, films, and devices by simply annealing to evaporate hydrazine, taking advantage of its low boiling point. In 2004 Mitzi et al. reported a technique for spin coating ultrathin (50 ̊̊) crystalline and continuous metal chalcogenide films, based on the low-temperature decomposition of highly soluble hydrazinium precursors [13]. A series of related thinfilm field-effect transistors (TFTs) have subsequently been fabricated using a similar strategy [19-21].

Moreover, mixed solvent systems have been demonstrated to be efficient media for the synthesis and controlled crystal growth of hybrid materials $[22,23]$, such as hybrid nanobelts [24], nanofibers [25], and nanoribbons [26]. These uniform nanostructured hybrid materials can be used as efficient precursors for fabricating pure inorganic nanostructured materials $[25,26]$.

Herein, we report a new approach for synthesizing hydrazine-cadmium tellurite hybrid microcrystals by a solvothermal reaction in a mixed solvent system and study their morphology evolution. These well-defined hydrazine-cadmium tellurite hybrid microcrystals can act as efficient precursors for the fabrication of porous semiconducting cadmium telluride and nanoporous tellurium architectures. The resulting porous cadmium telluride and tellurium not only retained similar external morphology on a micrometer or larger scale, but also displayed tailored internal nanostructures.

\section{Experimental}

Sodium tellurite $(99 \%)$, cadmium chloride hemidihydrate $(99 \%)$, hydrazine hydrate solution (85 wt.\%), n-propylamine (98\%), and hydrochloric acid (37 wt.\%) were all purchased from Shanghai Chemical Reagents
Co. Ltd. All chemicals were used directly as received without any purification.

\subsection{Hydrazine-cadmium tellurite hybrid microcry- stals grown in a mixed solvent system}

Typically, $0.5 \mathrm{mmol}$ of sodium tellurite, $0.5 \mathrm{mmol}$ of cadmium chloride hemidihydrate, $6 \mathrm{~mL}$ of n-propylamine, $3 \mathrm{~mL}$ of deionized water, and $2 \mathrm{~mL}$ of hydrazine hydrate were added to a Teflon-lined autoclave with a capacity of $23 \mathrm{~mL}$. The mixture was vigorously stirred magnetically for $10 \mathrm{~min}$ to form a homogeneous suspension. The autoclave was then sealed and heated at $100{ }^{\circ} \mathrm{C}$ for a certain period. After being cooled to room temperature, the grey powder was collected by centrifugation and washed three times each with ethanol and then distilled water. The final product was dried at $50{ }^{\circ} \mathrm{C}$ in a vacuum oven.

\subsection{Fabrication of porous cadmium telluride architecture}

Typically, $0.1 \mathrm{~g}$ of uniform hydrazine-cadmium tellurite hybrid microcrystals was transferred into a porcelain boat. The boat was put into a tube furnace and heated to $450{ }^{\circ} \mathrm{C}$ and kept for $2 \mathrm{~h}$ under a nitrogen atmosphere. After being cooled to room temperature naturally, the brown product was collected. This material was then added to $40 \mathrm{~mL}$ of $0.1 \mathrm{~mol} / \mathrm{L} \mathrm{HCl}$ solution and the mixture stirred for $10 \mathrm{~min}$. The resulting black powder was collected by centrifugation and washed three times each with deionized water and ethanol and finally dried at $50{ }^{\circ} \mathrm{C}$ in a vacuum oven. The mass of the final product was $0.0256 \mathrm{~g}$, corresponding to a yield of cadmium telluride of $51.30 \mathrm{wt} . \%$.

\subsection{Fabrication of porous tellurium architecture}

Typically, $0.1 \mathrm{~g}$ of uniform hydrazine-cadmium tellurite hybrid microcrystals was transferred into a porcelain boat. The boat was put into a tube furnace and heated to $300{ }^{\circ} \mathrm{C}$ and kept for $2 \mathrm{~h}$ under a nitrogen atmosphere. After being cooled to room temperature naturally, the brown product was collected. This material was then added to $40 \mathrm{~mL}$ of $0.1 \mathrm{~mol} / \mathrm{L} \mathrm{HCl}$ solution and the mixture stirred for $10 \mathrm{~min}$. The resulting black powder was collected by centrifugation and washed three times each with deionized water and ethanol 
and finally dried at $50{ }^{\circ} \mathrm{C}$ in a vacuum oven. The mass of the final product was $0.0176 \mathrm{~g}$, corrresponding to a yield of tellurium of 44.22 wt. $\%$.

\subsection{Characterization}

The phases of the as-prepared products were determined by powder X-ray diffraction (PXRD) using a Philips X'Pert Pro Super X-ray diffractometer equipped with graphite monochromatized $\mathrm{Cu} \mathrm{K \alpha}$ radiation $(\lambda=1.54178 \AA)$. Transmission electron microscopy (TEM) images were recorded with a Hitachi H-7650 transmission electron microscope at an acceleration voltage of $120 \mathrm{kV}$. Scanning electron microscopy (SEM) images were taken with a Zeiss Supra 40 scanning electron microscope at an acceleration voltage of $5 \mathrm{kV}$. Thermal gravimetric analysis (TGA) was carried out with a Shimadzu DTG-60H thermal analyzer, with a heating rate of $10^{\circ} \mathrm{C} / \mathrm{min}$ and an $\mathrm{N}_{2}$ flow of $50 \mathrm{~mL} / \mathrm{min}$. UV-visible spectra of the solid samples were recorded on a Shimadzu DUV-3700 spectrometer. BrunauerEmmett-Teller (BET) analysis was carried out on a Micromeritics ASAP $2020 \mathrm{M}+\mathrm{C}$ instrument. The inorganic elements were determined by inductively coupled plasma atomic emission spectroscopy (ICPAES). The composition of $\mathrm{N}, \mathrm{C}$, and $\mathrm{H}$ was determined using an Elementar Vario EL III analyzer.

\section{Results and discussion}

\subsection{Synthesis and formula determination of the hydrazine-cadmium tellurite hybrid microcrystals}

Hydrazine-cadmium tellurite hybrid microcrystals were obtained from a solvothermal reaction. The PXRD pattern of the product is shown in Fig. 1(a). There is no matching PXRD pattern in the powder diffraction file (PDF) database of the International Centre for Diffraction Data (ICDD), which indicates that this hybrid material is a totally new phase. The PXRD pattern was indexed by the semi-exhausive trial-and-error powder indexing (TREOR) method using the program Powder $X$ [27] which gave lattice para-meters of $a=7.97 \AA, b=9.32 \AA, c=7.78 \AA, \alpha=90^{\circ}$, $\beta=97.25^{\circ}$, and $\gamma=90^{\circ}$. The main indexed peaks are marked in Fig. 1(a) and the indexing of the diffraction peaks is given in Table S- 1 in the Electronic Supplementary Material (ESM).

Analysis of the PXRD pattern indicates that the product is possibly a hybrid material because of the large lattice parameters. In order to confirm the composition of the product, elemental analysis was carried out. The elements $\mathrm{N}, \mathrm{C}, \mathrm{H}, \mathrm{Cd}$, and Te in the sample were all detected but the amount of $C$ was very small (0.35 wt.\%), which indicates that the $n$-propylamine was only adsorbed on the surface of the sample instead of being incorporated into the hybrid structure. The sum of the mass percentages of $\mathrm{N}, \mathrm{H}, \mathrm{Cd}$, and Te was only $85.07 \mathrm{wt} . \%$. Furthermore, X-ray photoelectron spectroscopy (XPS) analysis indicated that the valence state of Te was mainly +4 (Fig. 1(b)). Hence, it can be concluded that oxygen was also present in the product. A possible formula for the product is $\mathrm{Cd}\left(\mathrm{N}_{2} \mathrm{H}_{4}\right) \mathrm{TeO}_{3}$, which is similar to our previously reported compounds of the type $\mathrm{Cd}(\mathrm{L}) \mathrm{TeO}_{3}$ ( $\mathrm{L}=$ a polyamine ) [25]. The calculated mass percentages of $\mathrm{N}, \mathrm{H}, \mathrm{Cd}$, and Te elements based on the proposed formula are given in Table 1 and are consistent with the experimental values. The following equation can be proposed for the thermal decomposition of $\mathrm{Cd}\left(\mathrm{N}_{2} \mathrm{H}_{4}\right) \mathrm{TeO}_{3}$ :

$3 \mathrm{Cd}\left(\mathrm{N}_{2} \mathrm{H}_{4}\right) \mathrm{TeO}_{3} \rightarrow 2 \mathrm{CdTe}+\mathrm{CdTeO}_{3}+3 \mathrm{~N}_{2}(\mathrm{~g})+6 \mathrm{H}_{2} \mathrm{O}(\mathrm{g})$

The TGA results further support the proposed formula. Figure 1(c) shows that the measured mass loss on heating the product is $19.12 \mathrm{wt} . \%$, which is consistent with the calculated mass loss for the above reaction of 19.99 wt.\%. The phases present after the TGA experiment were identified as a mixture of CdTe and $\mathrm{CdTeO}_{3}$ (Fig. 1(d)), which is also consistent with the proposed thermal decomposition process shown in Eq. (1).

Table 1 Elemental analysis results and the corresponding calculated values based on the proposed formula

\begin{tabular}{lccccc}
\hline \multicolumn{1}{c}{ Element } & $\mathrm{Cd}$ & $\mathrm{Te}$ & $\mathrm{N}$ & $\mathrm{H}$ & $\mathrm{C}$ \\
\hline Found (wt.\%) & 37.85 & 35.97 & 9.31 & 1.93 & 0.35 \\
Calculated (wt.\%) & 35.12 & 39.86 & 8.75 & 1.25 & 0 \\
\hline
\end{tabular}




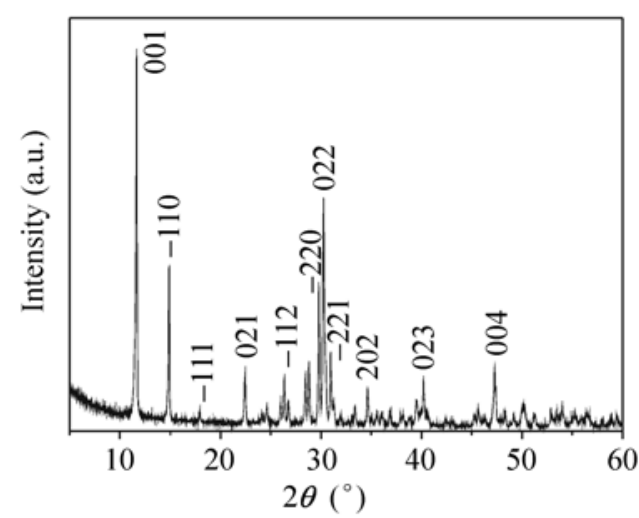

(a)

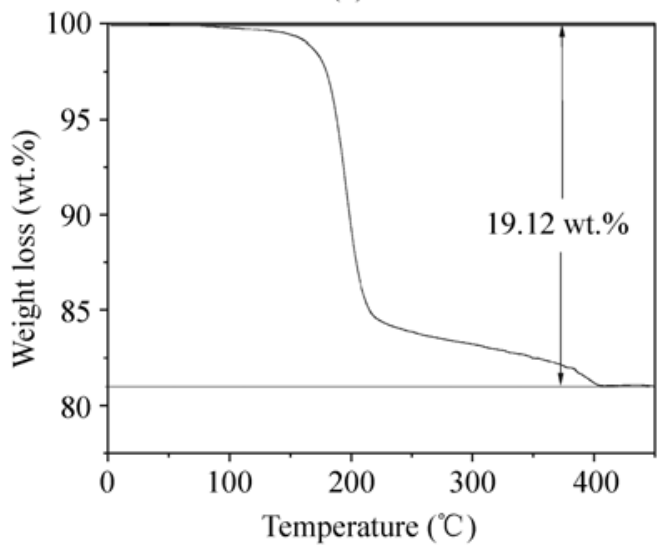

(c)

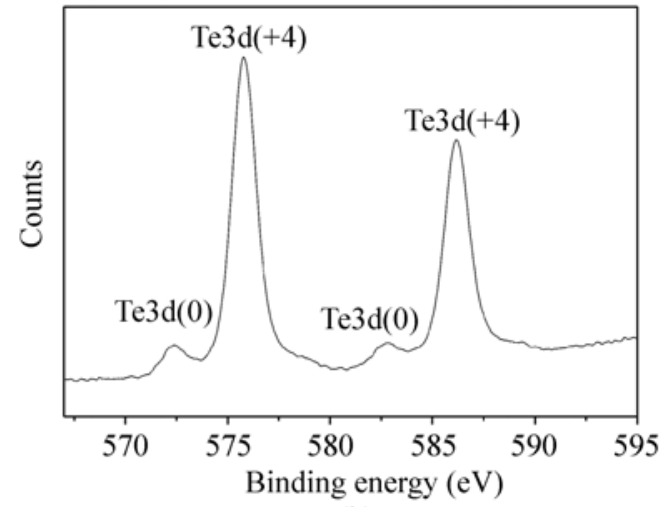

(b)

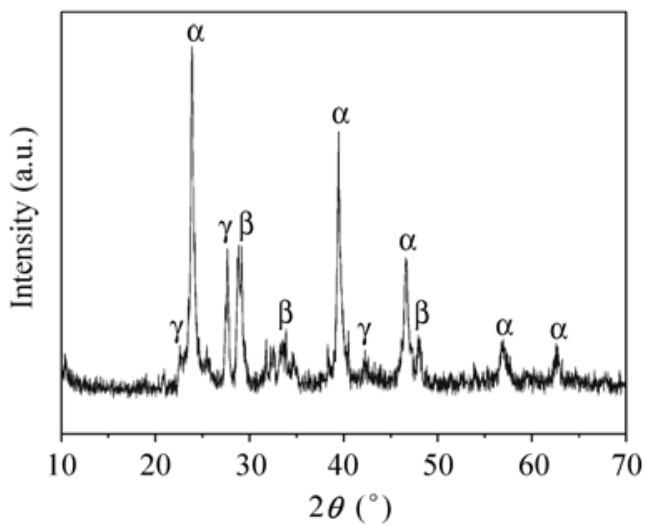

(d)

Figure 1 (a) PXRD pattern of the product of the solvothermal reaction. The pattern was indexed by the TREOR method using the program Powder X and the main indexed peaks are marked. (b) XPS spectrum of the Te $3 \mathrm{~d}$ region of the product of the solvothermal reaction. (c) TGA curve of the product of the solvothermal reaction. (d) PXRD pattern of the product after TGA ( $\alpha$ represents peaks of $\mathrm{CdTe}, \beta$ represents peaks of $\mathrm{CdTeO}_{3}$, and $\gamma$ represents peaks of Te impurity)

\subsection{Morphology evolution of $\mathrm{Cd}\left(\mathrm{N}_{2} \mathrm{H}_{4}\right) \mathrm{TeO}_{3}$ with increasing reaction time}

SEM images of as-synthesized $\mathrm{Cd}\left(\mathrm{N}_{2} \mathrm{H}_{4}\right) \mathrm{TeO}_{3}$ hybrid microcrystals are shown in Fig. 2. The hybrid microcrystals have a uniform platelet morphology with a mean lateral size of several micrometers.

The evolution of phases and morphologies of $\mathrm{Cd}\left(\mathrm{N}_{2} \mathrm{H}_{4}\right) \mathrm{TeO}_{3}$ hybrid microcrystals grown in the mixed solvent system were investigated by time-dependent experiments. Figure 3 shows the PXRD patterns of products obtained after different reaction times. The

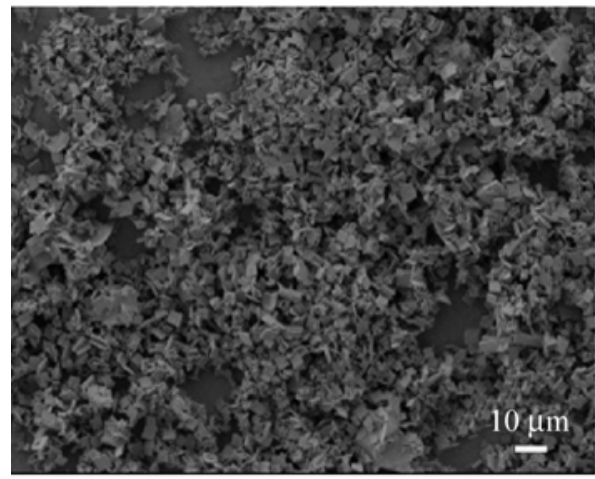

(a)

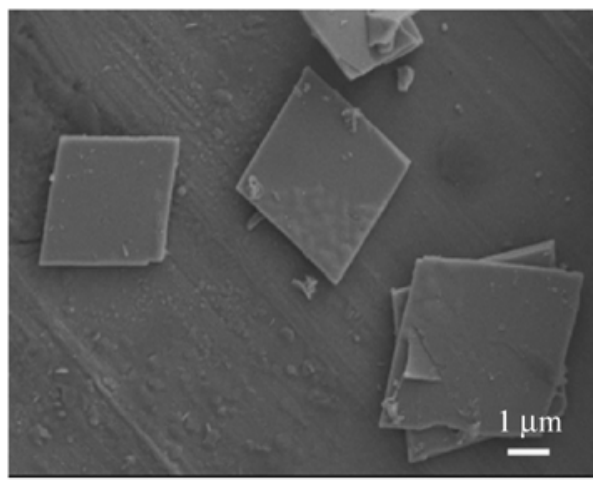

(b)

Figure 2 SEM images of $\mathrm{Cd}\left(\mathrm{N}_{2} \mathrm{H}_{4}\right) \mathrm{TeO}_{3}$ hybrid microcrystals: (a) low magnification image and (b) higher magnification image 


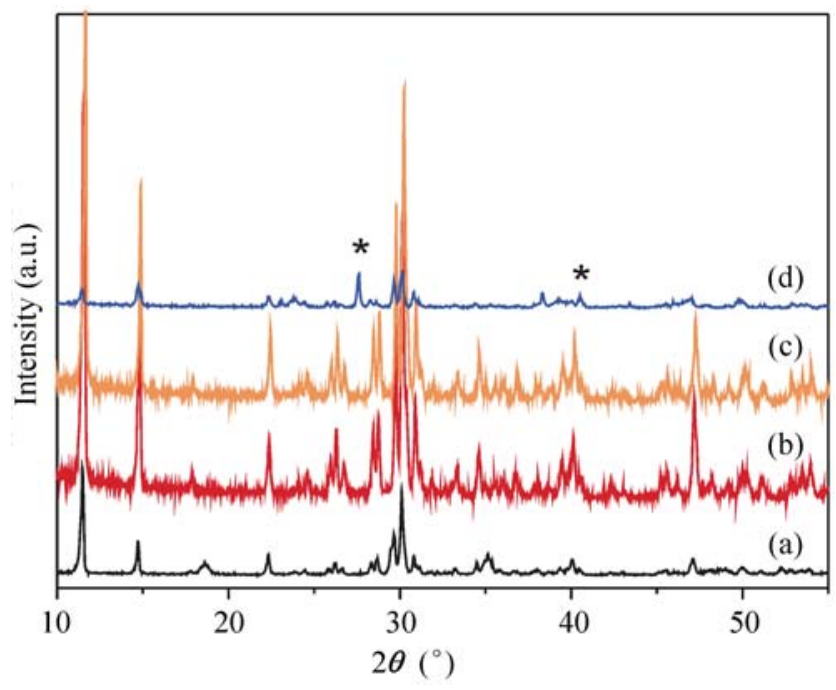

Figure 3 PXRD patterns of the product obtained in a mixed solvent system with different reaction time: (a) $12 \mathrm{~h}$, (b) 2 days, (c) 5 days, and (d) 7 days. * denotes a Te phase

intensities of the diffraction peaks varied with reaction time, but the the main phases were always present. At the initial reaction stage, the intensity of the diffraction peaks was low (Fig. 3(a)), and became higher with increasing reaction time (Figs. 3(b) and 3(c)). However, the peak intensities decreased again with very long reaction time and a small amount of a new phase, Te, was formed (Fig. 3(d)).

The variation in diffraction peak intensity is related to the evolution of morphology with reaction time. SEM images of the hybrid microcrystals produced at different reaction time are shown in Fig. 4. Plateletlike crystals were initially formed (Fig. 4(a)), and then other hybrid crystals grew on the surface of the initially formed platelet-like crystals when the reaction time was prolonged (Fig. 4(b)) and the crystallinity was further improved (which is consistent with the observed increase in the diffraction peak intensities). However, further increases in reaction time led to the collapse of the hybrid crystals (see the inset image in Fig. 4(c)), which can be attributed to the reaction of the hydrazine in the hybrid structure with the cadmium tellurite. Finally, the morphologies of the hybrid crystals evolved into the hierarchical structures shown in Fig. 4(d) as result of the collapse of the hybrid crystals due to the reaction between hydrazine and cadmium tellurite. The reduction in the intensity of the diffraction peaks of the hybrid phase and the appearance of new
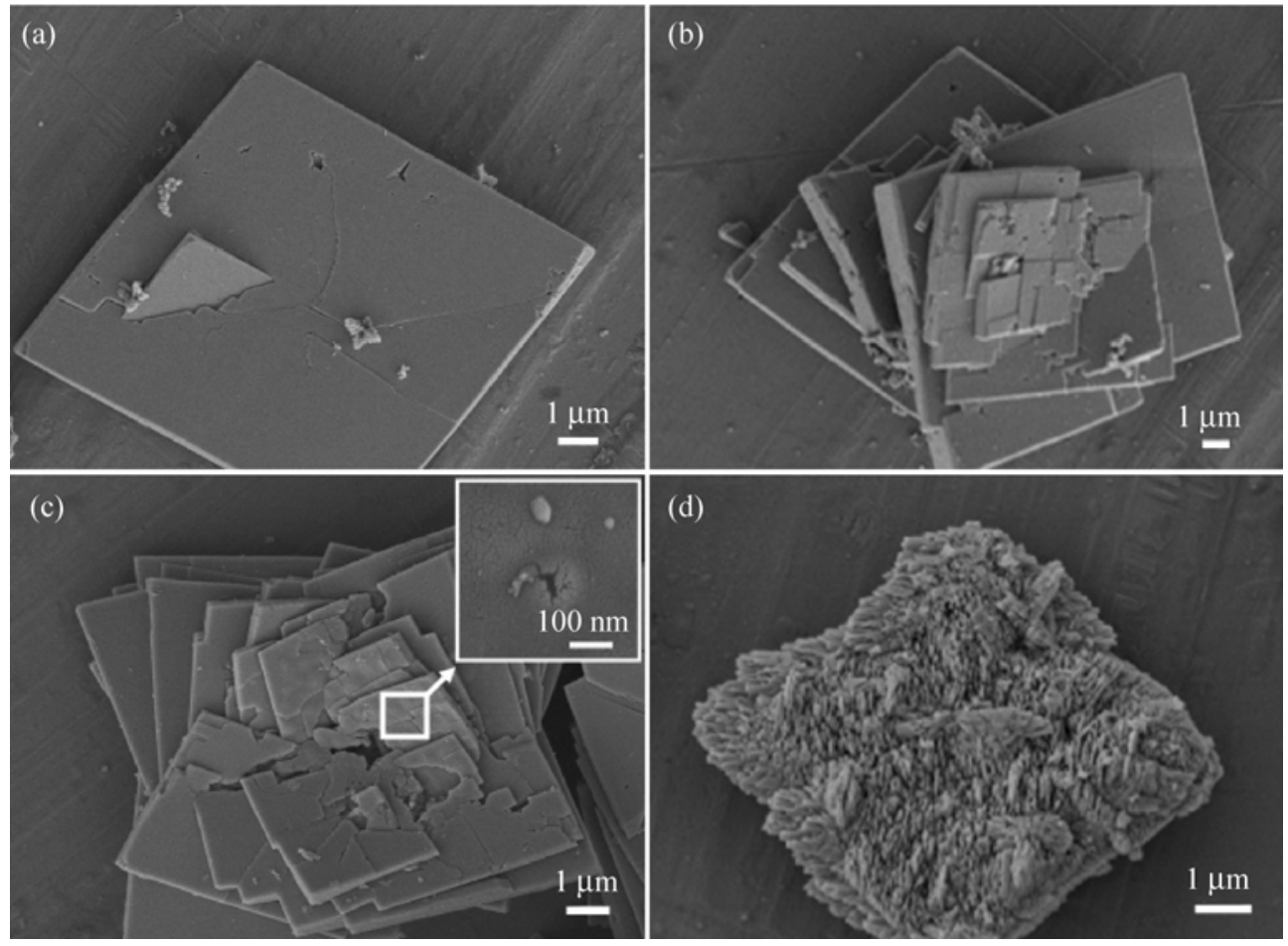

Figure 4 SEM images of hybrid microcrystals formed after different reaction time: (a) 12 h, (b) 2 days, (c) 5 days, and (d) 7 days. The inset image in (c) shows the area where dissolution of the crystal is occurring 
peaks corresponding to a Te phase (Fig. 3(d)) further confirmed the reaction between the hydrazine and cadmium tellurite at extended reaction times.

\subsection{Fabrication of porous architectures by temperature-dependent thermal decomposition}

As discussed above, the proposed stoichiometry of the hydrazine-cadmium tellurite hybrid microcrystals is $\mathrm{Cd}\left(\mathrm{N}_{2} \mathrm{H}_{4}\right) \mathrm{TeO}_{3}$. TGA indicated a very rapid mass loss in the range $150-450{ }^{\circ} \mathrm{C}$ which can be attributed to the elimination of hydrazine. It should be very interesting to study the evolution of morphologies and phase transformations during the thermal decomposition process. Since hydrazine is a very volatile material and a strong reducing agent, its vaporization should lead to highly porous structures as has previously been reported when $\mathrm{CO}_{2}$ gas was evolved [28, 29].

A systematic investigation of the thermal decomposition of $\mathrm{Cd}\left(\mathrm{N}_{2} \mathrm{H}_{4}\right) \mathrm{TeO}_{3}$ was carried out. The PXRD patterns of the thermal decomposition products obtained at different annealing temperatures are shown in Fig. 5. An almost amorphous phase of $\mathrm{Cd}\left(\mathrm{N}_{2} \mathrm{H}_{4}\right) \mathrm{TeO}_{3}$ was first formed (Fig. 5(a)) due to some elimination of hydrazine from the hybrid structure. Crystalline CdTe was then formed at higher thermal decomposition temperature (Fig. 5(b)), as a result of reduction by hydrazine. Thus, on increasing the thermal decomposition temperature, the almost amorphous $\mathrm{Cd}\left(\mathrm{N}_{2} \mathrm{H}_{4}\right) \mathrm{TeO}_{3}$ phase was transformed first to $\mathrm{CdTeO}_{3}$ and finally a mixture of $\mathrm{CdTe}$ and $\mathrm{CdTeO}_{3}$ was obtained (Fig. 5(e)); this is consistent with the proposed thermal decomposition process shown in Eq. (1). Figure 6 shows SEM images of thermal decomposition products obtained at different annealing temperatures. When the $\mathrm{Cd}\left(\mathrm{N}_{2} \mathrm{H}_{4}\right) \mathrm{TeO}_{3}$ platelet-like microcrystals were heated to $300{ }^{\circ} \mathrm{C}$ and kept at that temperature for $2 \mathrm{~h}$, only a few pores appeared on the surfaces of the hybrid microcrystals (Fig. 6(a)). When the thermal decomposition temperature was increased to $350{ }^{\circ} \mathrm{C}$, the morphologies changed relatively little, but more pores and cracks appeared on the surfaces (Fig. 6(b)).

A drastic morphology transformation occurred at $400{ }^{\circ} \mathrm{C}$ as shown in Fig. 6(c). The microcrystals became

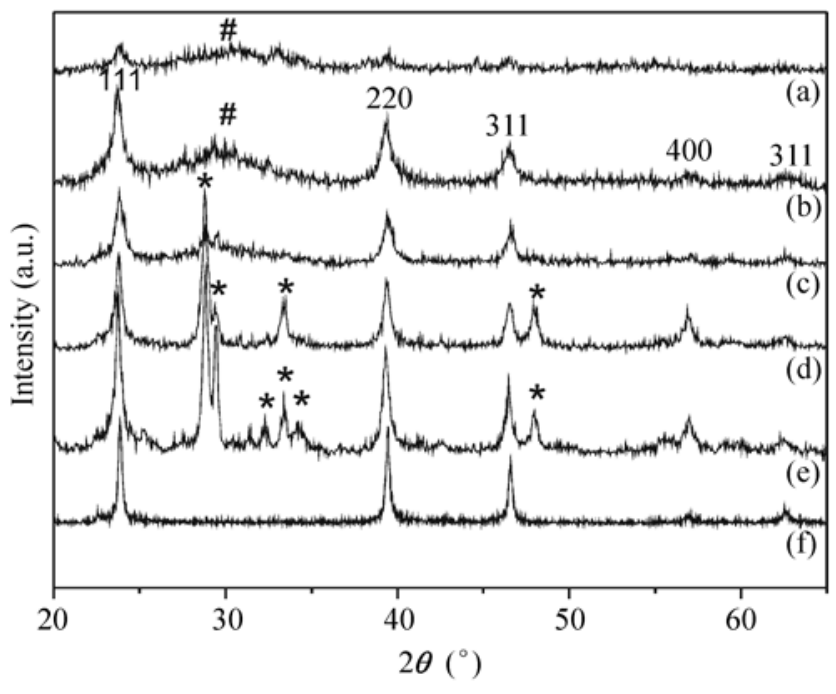

Figure 5 PXRD patterns of the thermal decomposition products of $\mathrm{Cd}\left(\mathrm{N}_{2} \mathrm{H}_{4}\right) \mathrm{TeO}_{3}$ at different temperatures: (a) $200^{\circ} \mathrm{C}$, (b) $300^{\circ} \mathrm{C}$, (c) $350^{\circ} \mathrm{C}$, (d) $400{ }^{\circ} \mathrm{C}$, and (e) $450^{\circ} \mathrm{C}$. (f) The PXRD pattern of porous $\mathrm{CdTe}$ architecture obtained by using hydrochloric acid to wash the sample obtained after thermal decomposition at $450^{\circ} \mathrm{C}$; the weak peak at ca. $23^{\circ}$ is the (001) diffraction peak of wurtzite phase CdTe. \# denotes amorphous $\mathrm{Cd}\left(\mathrm{N}_{2} \mathrm{H}_{4}\right) \mathrm{TeO}_{3}$. * denotes $\mathrm{CdTeO}_{3}$

porous and pores appeared inside the microcrytals (inset image in Fig. 6(c)). The PXRD pattern also displayed significant changes in that peaks from the almost amorphous $\mathrm{Cd}\left(\mathrm{N}_{2} \mathrm{H}_{4}\right) \mathrm{TeO}_{3}$ disappeared, but those from crystalline $\mathrm{CdTeO}_{3}$ appeared (Fig. 5(d)). With a further increase in annealing temperature to $450{ }^{\circ} \mathrm{C}$, the pores shrank and collapsed, resulting in the formation of porous structures (Fig. 6(d)). PXRD shows that these porous microcrystals were a mixture of $\mathrm{CdTe}$ and $\mathrm{CdTeO}_{3}$ (Fig. 5(e)). Hydrochloric acid was introduced to dissolve the $\mathrm{CdTeO}_{3}$ phase in the porous architectures, leading to pure porous $\mathrm{CdTe}$ (Fig. 5(f)). The resulting porous CdTe microcrystals still retained a platelet-like morphology as shown in Fig. 7.

\subsection{Fabrication of porous tellurium architectures through hydrochloric acid leaching}

Figure $5(b)$ shows that the hybrid $\mathrm{Cd}\left(\mathrm{N}_{2} \mathrm{H}_{4}\right) \mathrm{TeO}_{3}$ material was transformed to a mixture of a $\mathrm{CdTe}$ phase with low crystallinity and amorphous $\mathrm{Cd}\left(\mathrm{N}_{2} \mathrm{H}_{4}\right) \mathrm{TeO}_{3}$ after thermal decomposition at $300{ }^{\circ} \mathrm{C}$. A dilute solution of hydrochloric acid was used to treat the thermally decomposed sample. Surprisingly, 

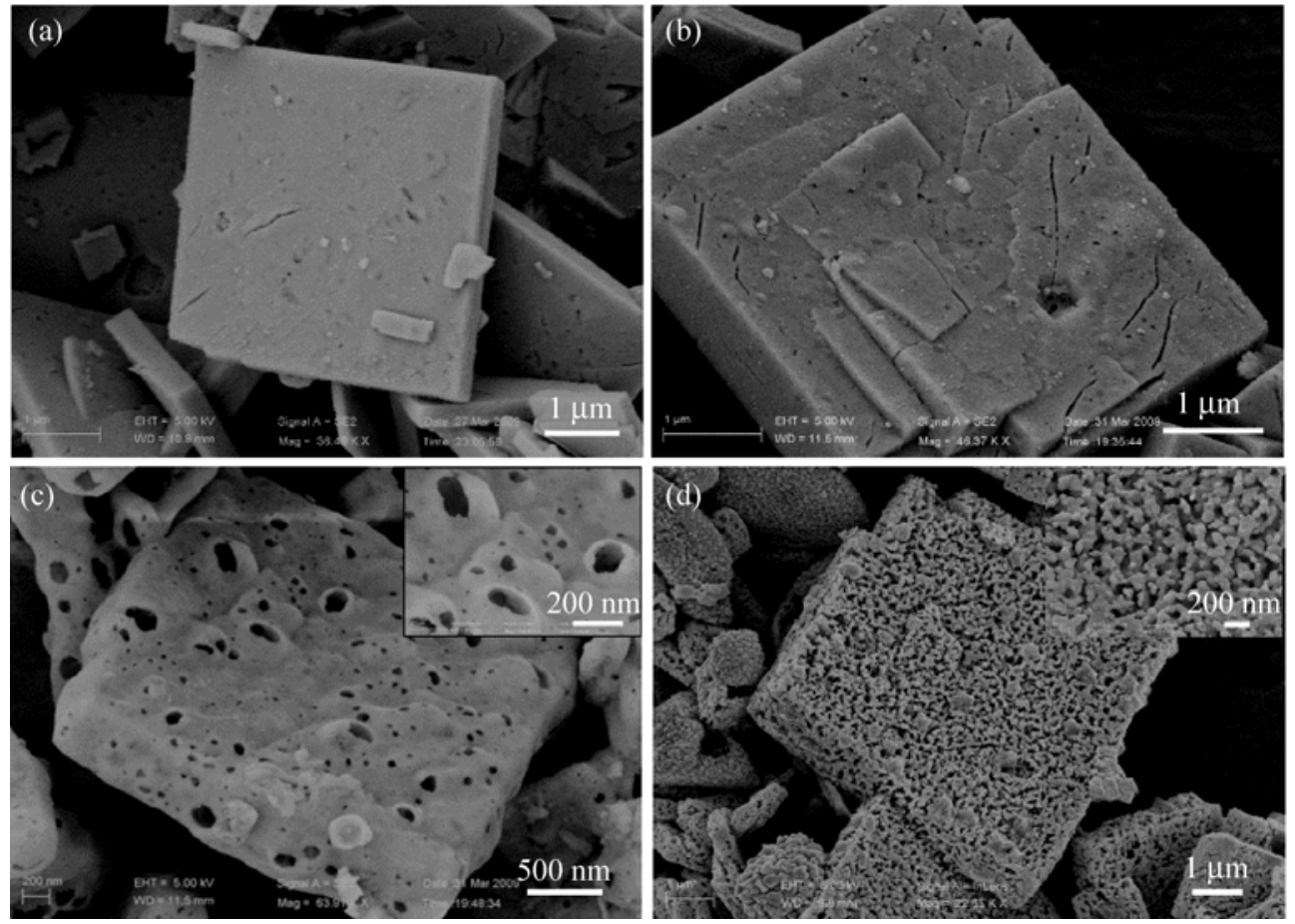

Figure 6 SEM images of $\mathrm{Cd}\left(\mathrm{N}_{2} \mathrm{H}_{4}\right) \mathrm{TeO}_{3}$ hybrid microcrystals thermally decomposed at different temperatures: (a) $300^{\circ} \mathrm{C}$, (b) $350^{\circ} \mathrm{C}$, (c) $400^{\circ} \mathrm{C}$, and (d) $450^{\circ} \mathrm{C}$. The inset images in (c) and (d) are at higher magnification

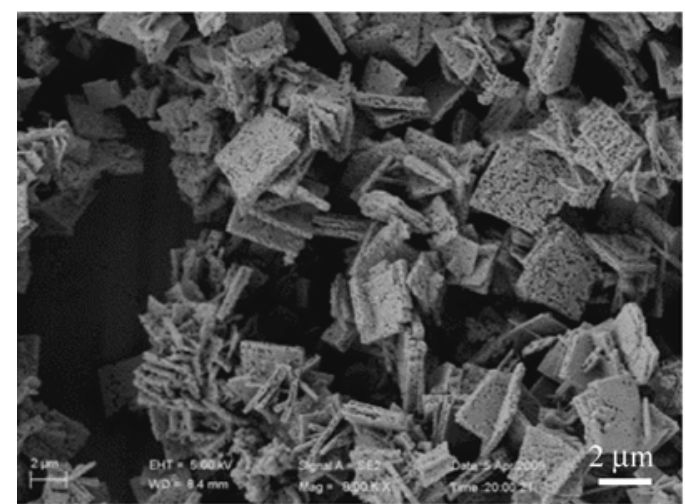

(a)

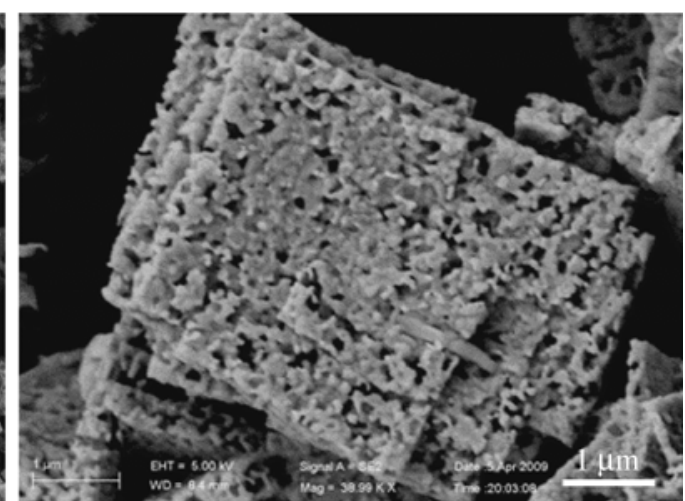

(b)

Figure 7 SEM images of porous CdTe microcrystals obtained by using hydrochloric acid to wash the sample obtained by thermal decomposition of $\mathrm{Cd}\left(\mathrm{N}_{2} \mathrm{H}_{4}\right) \mathrm{TeO}_{3}$ at $450{ }^{\circ} \mathrm{C}$ : (a) low-magnification image and (b) higher-magnification image

the sample was converted to a pure tellurium phase (Fig. 8(a)) and pores were formed inside the microcrystals (Figs. 8(b) and 9). Both TEM and SEM images showed that the porous microcrystals retained the original platelet-like shape. The pores were homogenously distributed throughout the microcrystals leading to a nanoporous architecture. To the best of our knowledge, this is the first report of the synthesis of self-supporting nanoporous tellurium microcrystals.
Interestingly, it was observed that subsequent washing with dilute hydrochloric acid for samples that had been thermally decomposed at different annealing temperatures afforded different phases and porous morphologies. When the hydrazinecadmium tellurite hybrid microcrystals were annealed at $300{ }^{\circ} \mathrm{C}$ for $2 \mathrm{~h}$, the material became amorphous because of elimination of hydrazine and poorly crystalline CdTe nanocrystals were formed by the action of hydrazine as a reducing agent (Fig. 5(b)). 


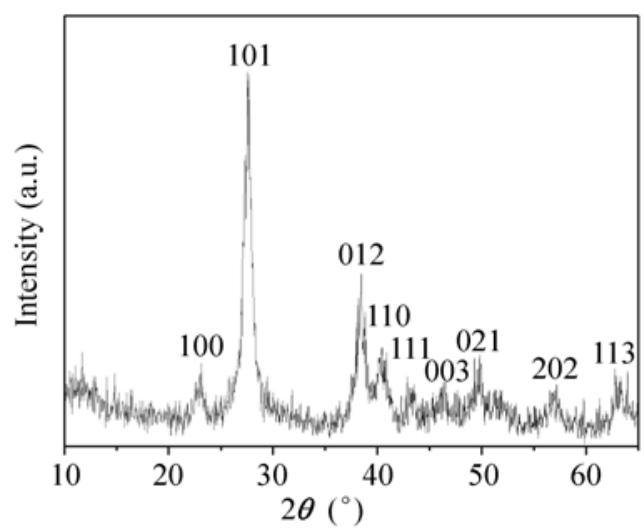

(a)

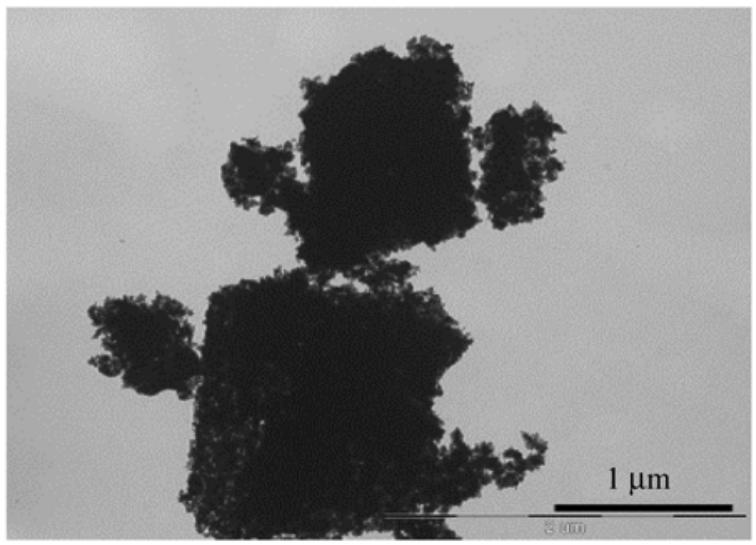

(b)

Figure 8 (a) PXRD pattern and (b) TEM image of the material obtained after thermal decomposition of $\mathrm{Cd}\left(\mathrm{N}_{2} \mathrm{H}_{4}\right) \mathrm{TeO}_{3}$ at $300{ }^{\circ} \mathrm{C}$ followed by treatment with dilute hydrochloric acid
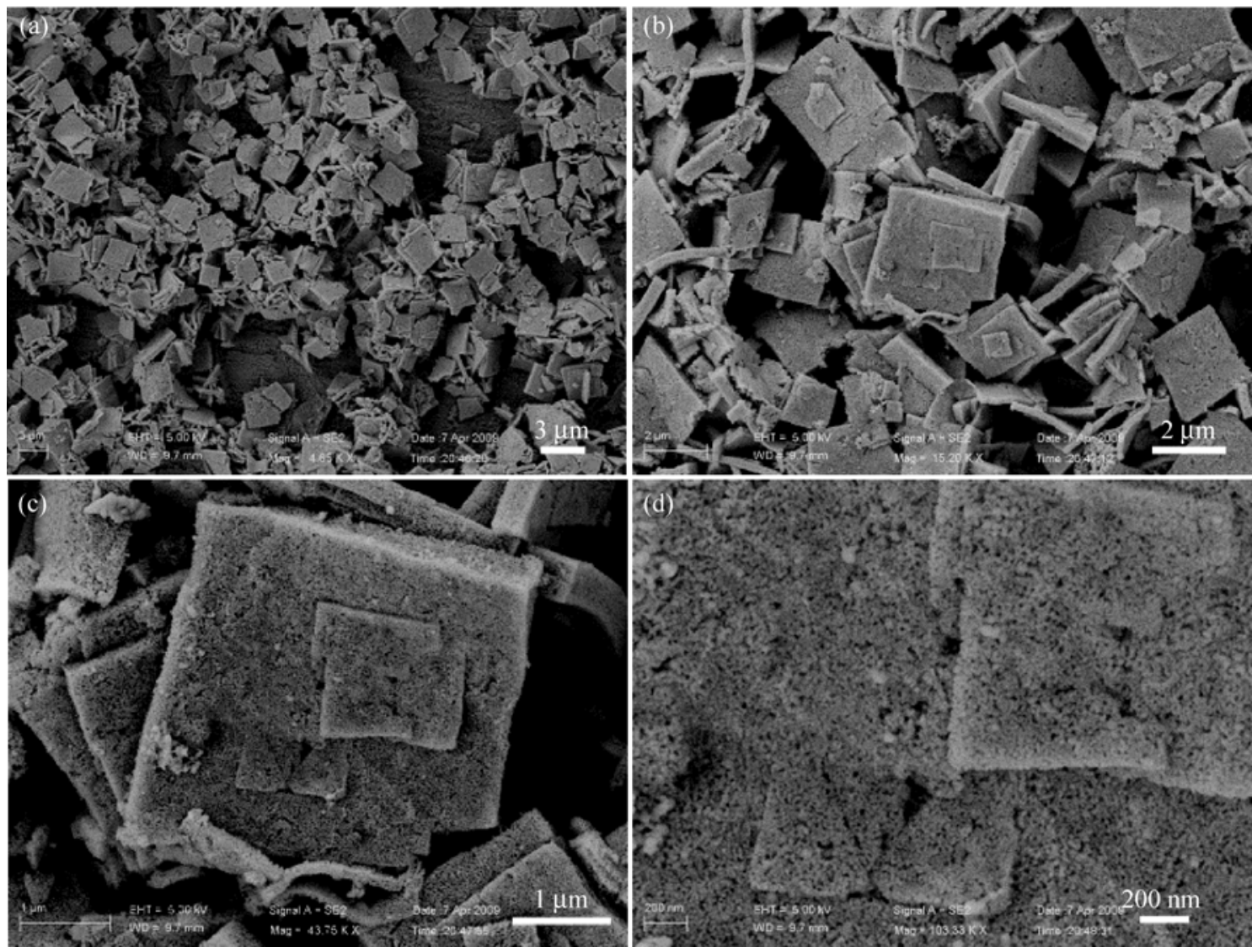

Figure 9 SEM images of nanoporous tellurium architectures obtained after thermal decomposition of $\mathrm{Cd}_{(}\left(\mathrm{N}_{2} \mathrm{H}_{4}\right) \mathrm{TeO}_{3}$ at $300^{\circ} \mathrm{C}$ followed by treatment with dilute hydrochloric acid

The sample obtained after thermal decomposition was brown. After adding this brown powder to dilute hydrochloric acid and stirring the suspension for several minutes, a black powder was formed. In this process, the amorphous $\mathrm{Cd}\left(\mathrm{N}_{2} \mathrm{H}_{4}\right) \mathrm{TeO}_{3}$ was easily dissolved by $\mathrm{HCl}$ and the residual three-dimensional skeleton of CdTe nanocrystallites-highly reactive by virtue of its low crystallinity-reacted spontaneously in situ with $\mathrm{TeO}_{3}{ }^{2-}$ to form the nanoporous tellurium architecture. This topotactic reaction can be expressed 
as follows:

$$
2 \mathrm{CdTe}+\mathrm{TeO}_{3}{ }^{2-}+6 \mathrm{H}^{+} \longrightarrow 3 \mathrm{Te}+2 \mathrm{Cd}^{2+}+3 \mathrm{H}_{2} \mathrm{O}
$$

Because the nanoporous tellurium architecture was formed under ambient conditions, its crystallinity was poor (Fig. 8(a)). The nanoporous architecture was also unstable under irradiation by the electron beam in TEM (see Fig. S-1 in the ESM).

When the hydrazine-cadmium tellurite hybrid microcrystals were annealed at $450{ }^{\circ} \mathrm{C}$ for $2 \mathrm{~h}$, the material was converted to a mixture of highly crystalline CdTe and $\mathrm{CdTeO}_{3}$ (Fig. 5(e)). On treatment of the product with dilute hydrochloric acid, the $\mathrm{CdTeO}_{3}$ phase dissolved leaving a porous $\mathrm{CdTe}$ architecture. The CdTe was relatively unreactive because of its high crystallinity and, unlike the earlier case, did not react with $\mathrm{TeO}_{3}{ }^{2-}$. Based on the above analysis and results, the complete transformation pathway from hydrazine-cadmium tellurite hybrid microcrystals to porous cadmium telluride and tellurium architectures can be proposed, as illustrated in Scheme 1.

\subsection{BET analysis and optical properties of porous cadmium telluride and tellurium architectures}

Nitrogen adsorption and desorption isotherms were obtained in order to determine the surface area and porosity of the porous cadmium telluride and tellurium architectures. Figures 10(a) and 10(b) show the nitrogen adsorption-desorption isotherms of the porous cadmium telluride and tellurium, respectively. The BET (Brunauer-Emmett-Teller) surface areas of

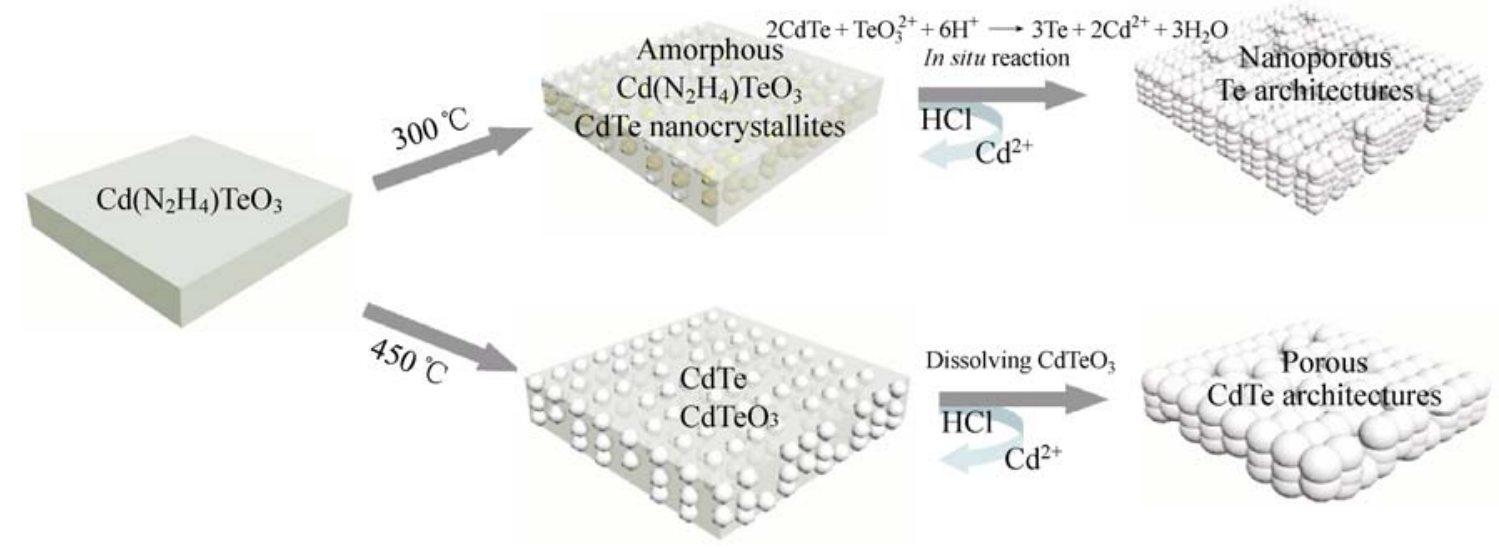

Scheme 1 Illustration of transformation pathways from hydrazine-cadmium tellurite hybrid microcrystals to porous tellurium and cadmium telluride architectures

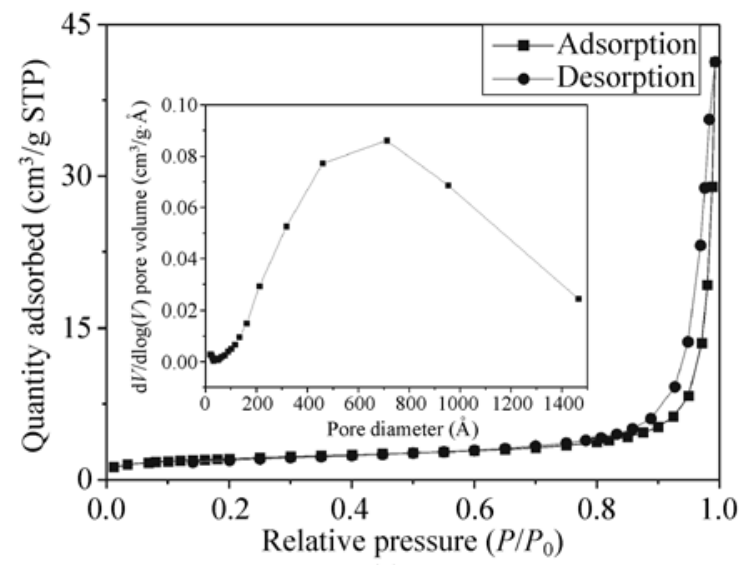

(a)

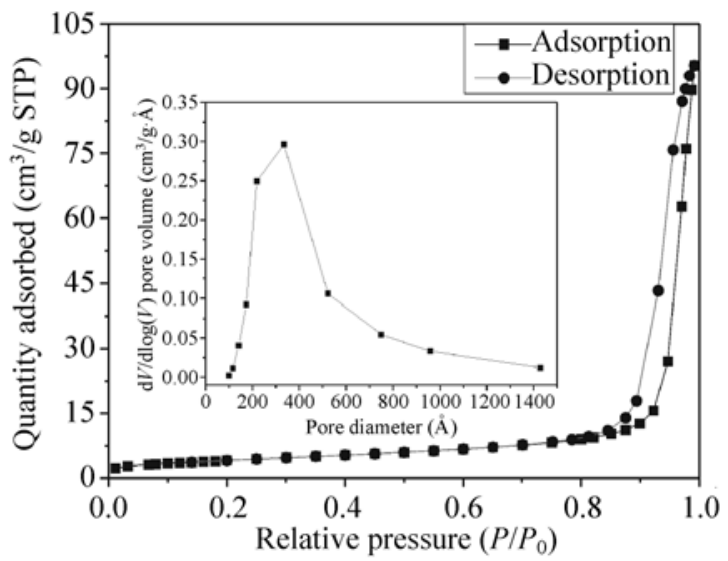

(b)

Figure 10 Nitrogen adsorption-desorption isotherms of (a) porous cadmium telluride and (b) nanoporous tellurium. The inserts show the corresponding pore size distributions

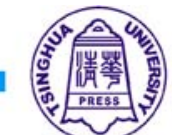


cadmium telluride and tellurium are $7.05 \mathrm{~m}^{2} / \mathrm{g}$ and $14.96 \mathrm{~m}^{2} / \mathrm{g}$, respectively, and the BJH (Barrett-JoynerHalenda) average pore diameters are about $70 \mathrm{~nm}$ and $40 \mathrm{~nm}$, respectively. The surface area and pore size of nanoporous tellurium are both better than the corresponding values for porous cadmium telluride, which is consistent with the SEM images. This is the first time that nanoporous semiconducting cadmium telluride and tellurium architectures have been fabricated by thermal decomposition of a hydrazinecadmium tellurite hybrid material.

The optical properties of the samples were studied by UV-visible spectroscopy, which showed that the band gap of the hydrazine-based hybrid materials was significantly different from those of porous cadmium telluride and tellurium. The absorption edge of the $\mathrm{Cd}\left(\mathrm{N}_{2} \mathrm{H}_{4}\right) \mathrm{TeO}_{3}$ hybrid occurs at about $300 \mathrm{~nm}$ (Fig. 11(b)), but the absorption edge of the porous $\mathrm{CdTe}$ architectures was red-shifted to $850 \mathrm{~nm}$. The UV-visible spectra of both porous CdTe and Te are similar to those of the corresponding bulk materials.

\section{Conclusions}

A well-defined platelet-like hydrazine-cadmium tellurite hybrid material can be synthesized by a facile reaction under mild conditions in a mixed solvent. The resulting hybrid material was fully characterized by PXRD, elemental analysis, TGA, and XPS; the results were consistent with the formula $\mathrm{Cd}\left(\mathrm{N}_{2} \mathrm{H}_{4}\right) \mathrm{TeO}_{3}$.

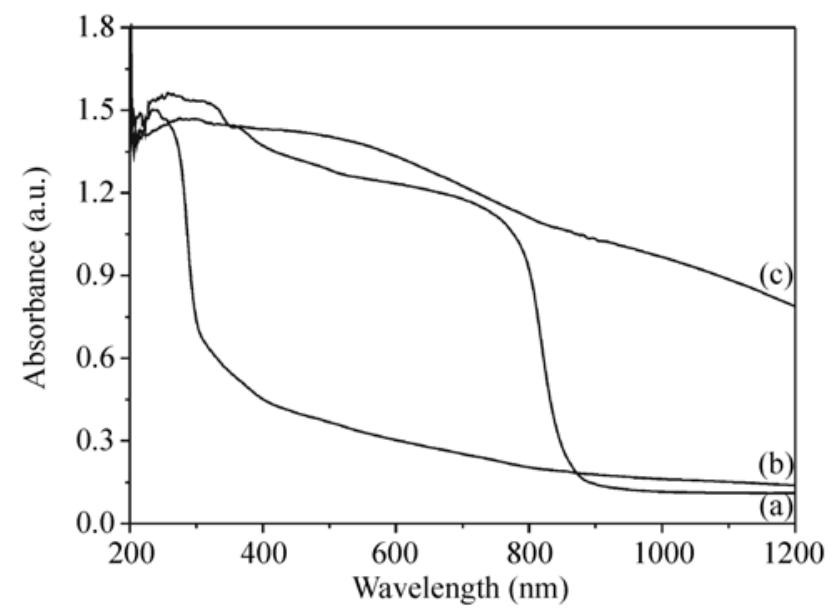

Figure 11 UV-visible spectra of (a) porous cadmium telluride, (b) $\mathrm{Cd}\left(\mathrm{N}_{2} \mathrm{H}_{4}\right) \mathrm{TeO}_{3}$, and (c) nanoporous tellurium
Control over the morphology and evolution of $\mathrm{Cd}\left(\mathrm{N}_{2} \mathrm{H}_{4}\right) \mathrm{TeO}_{3}$ with reaction time have been discussed. Because of the high volatility and reducing capacity of hydrazine, $\mathrm{Cd}\left(\mathrm{N}_{2} \mathrm{H}_{4}\right) \mathrm{TeO}_{3}$ undergoes a temperaturedependent thermal decomposition. It was found that porous cadmium telluride and nanoporous tellurium architectures can be selectively produced by treating the samples formed by thermal decomposition at different temperatures with hydrochloric acid. BET analysis and a study of the optical properties of the porous samples are also reported. The as-synthesized porous cadmium telluride and tellurium microcrystals have potential uses in sensor devices or as templates for the synthesis of other porous materials.

\section{Acknowledgements}

This work was supported by the National Basic Research Priorities Program of China (No. 2010CB934700), the National Natural Science Foundation of China (NSFC) (Nos. 50732006 and 20671085), the Program of International $S$ and $T$ Cooperation (No. S2010GR0314), and the PartnerGroup of the Chinese Academy of Sciences-the Max Planck Society.

Electronic Supplementary Material: An additional TEM image and indexing of the PXRD data are available in the online version of this article at http://dx.doi.org/10.1007/s12274-010-1011-7 and are accessible free of charge.

\section{References}

[1] Sanchez, C.; Julian, B.; Belleville, P.; Popall, M. Applications of hybrid organic-inorganic nanocomposites. J. Mater. Chem. 2005, 15, 3559-3592.

[2] Huang, X. Y.; Li, J.; Fu, H. X. The first covalent organicinorganic networks of hybrid chalcogenides: Structures that may lead to a new type of quantum wells. J. Am. Chem. Soc. 2000, 122, 8789-8790.

[3] Heulings, H. R.; Huang, X. Y.; Li, J.; Yuen, T.; Lin, C. L. Mn-substituted inorganic-organic hybrid materials based on ZnSe: Nanostructures that may lead to magnetic semiconductors with a strong quantum confinement effect. Nano Lett. 2001, 1, 521-525.

[4] Huang, X. Y.; Heulings, H. R.; Le, V.; Li, J. Inorganic-organic 
hybrid composites containing MQ ( II-VI) slabs: A new class of nanostructures with strong quantum confinement and periodic arrangement. Chem. Mater. 2001, 13, 3754-3759.

[5] Huang, X. Y.; Li, J.; Zhang, Y.; Mascarenhas, A. From 1D chain to 3D network: Tuning hybrid II-VI nanostructures and their optical properties. J. Am. Chem. Soc. 2003, 125, 7049-7055.

[6] Fluegel, B.; Zhang, Y.; Mascarenhas, A.; Huang, X.; Li, J. Electronic properties of hybrid organic-inorganic semiconductors. Phys. Rev. B 2004, 70, 205308.

[7] Fu, H. X.; Li, J. Density-functional study of organic-inorganic hybrid single crystal $\mathrm{ZnSe}\left(\mathrm{C}_{2} \mathrm{H}_{8} \mathrm{~N}_{2}\right)_{1 / 2}$. J. Chem. Phys. 2004, 120, 6721-6725.

[8] Zhang, Y.; Dalpian, G. M.; Fluegel, B.; Wei, S. H.; Mascarenhas, A.; Huang, X. Y.; Li, J.; Wang, L. W. Novel approach to tuning the physical properties of organicinorganic hybrid semiconductors. Phys. Rev. Lett. 2006, 96, 026405 .

[9] Huang, X. Y.; Li, J. From single to multiple atomic layers: A unique approach to the systematic tuning of structures and properties of inorganic-organic hybrid nanostructured semiconductors. J. Am. Chem. Soc. 2007, 129, 3157-3162.

[10] Li, J.; Bi, W. H.; Ki, W.; Huang, X. Y.; Reddy, S. Nanostructured crystals: Unique hybrid semiconductors exhibiting nearly zero and tunable uniaxial thermal expansion behavior. J. Am. Chem. Soc. 2007, 129, 14140-14141.

[11] Zhang, Y.; Islam, Z.; Ren, Y.; Parilla, P. A.; Ahrenkiel, S. P.; Lee, P. L.; Mascarenhas, A.; McNevin, M. J.; Naumov, I.; Fu, H. X.; Huang, X. Y.; Li, J. Zero thermal expansion in a nanostructured inorganic-organic hybrid crystal. Phys. Rev. Lett. 2007, 99, 215901.

[12] Ki, W.; Li, J. A semiconductor bulk material that emits direct white light. J. Am. Chem. Soc. 2008, 130, 8114-8115.

[13] Mitzi, D. B.; Kosbar, L. L.; Murray, C. E.; Copel, M.; Afzali, A. High-mobility ultrathin semiconducting films prepared by spin coating. Nature 2004, 428, 299-303.

[14] Mitzi, D. B. Synthesis, structure, and thermal properties of soluble hydrazinium germanium(IV) and tin(IV) selenide salts. Inorg. Chem. 2005, 44, 3755-3761.

[15] Mitzi, D. B. Polymorphic one-dimensional $\left(\mathrm{N}_{2} \mathrm{H}_{4}\right)_{2} \mathrm{ZnTe}$ : Soluble precursors for the formation of hexagonal or cubic zinc telluride. Inorg. Chem. 2005, 44, 7078-7086.

[16] Mitzi, D. B. $\mathrm{N}_{4} \mathrm{H}_{9} \mathrm{Cu}_{7} \mathrm{~S}_{4}$ : A hydrazinium-based salt with a layered $\mathrm{Cu}_{7} \mathrm{~S}_{4}^{-}$framework. Inorg. Chem. 2007, 46, 926-931.

[17] Yuan, M.; Dirmyer, M.; Badding, J.; Sen, A.; Dahlberg, M.; Schiffer, P. Controlled assembly of zero-, one-, two-, and three-dimensional metal chalcogenide structures. Inorg. Chem. 2007, 46, 7238-7240.

[18] Manos, M. J.; Kanatzidis, M. G. Use of hydrazine in the hydrothermal synthesis of chalcogenides: The neutral framework material $\left[\mathrm{Mn}_{2} \mathrm{SnS}_{4}\left(\mathrm{~N}_{2} \mathrm{H}_{4}\right)_{2}\right]$. Inorg. Chem. 2009, 48, 4658-4660.

[19] Mitzi, D. B.; Copel, M.; Chey, S. J. Low-voltage transistor employing a high-mobility spin-coated chalcogenide semiconductor. Adv. Mater. 2005, 17, 1285-1289.

[20] Milliron, D. J.; Mitzi, D. B.; Copel, M.; Murray, C. E. Solution-processed metal chalcogenide films for p-type transistors. Chem. Mater. 2006, 18, 587-590.

[21] Mitzi, D. B. Solution-processed inorganic semiconductors. J. Mater. Chem. 2004, 14, 2355-2365.

[22] Yao, W. T.; Yu, S. H. Synthesis of semiconducting functional materials in solution: From II-VI semiconductor to inorganic-organic hybrid semiconductor nanomaterials. $A d v$. Funct. Mater. 2008, 18, 3357-3366.

[23] Yao, H. B.; Gao, M. R.; Yu, S. H. Small organic molecule templating synthesis of organic-inorganic hybrid materials: Their nanostructures and properties. Nanoscale 2010, DOI:10.1039/B9NR00192A.

[24] Yao, W. T.; Yu, S. H.; Huang, X. Y.; Jang, J.; Zhao, L. Q. Pan, L.; Li, J. Nanocrystals of an inorganic-organic hybrid semiconductor: Formation of uniform nanobelts of [ZnSe] (diethylenetriamine $)_{0.5}$ in a ternary solution. Adv. Mater. 2005, 17, 2799-2802.

[25] Yao, H. B.; Li, X. B.; Yu, S. H. New blue-light-emitting ultralong $\left[\mathrm{Cd}(\mathrm{L})\left(\mathrm{TeO}_{3}\right)\right](\mathrm{L}=$ polyamine$)$ organic-inorganic hybrid nanofiber bundles: Their thermal stability and acidic sensitivity. Chem. Eur. J. 2009, 15, 7611-7618.

[26] Zang, Z. A.; Yao, H. B.; Zhou, Y. X.; Yao, W. T.; Yu, S. H. Synthesis and magnetic properties of new $\left[\mathrm{Fe}_{18} \mathrm{~S}_{25}\right](\mathrm{TETAH})_{14}$ (TETAH $=$ protonated triethylenetetramine) nanoribbons: An efficient precursor to $\mathrm{Fe}_{7} \mathrm{~S}_{8}$ nanowires and porous $\mathrm{Fe}_{2} \mathrm{O}_{3}$ nanorods. Chem. Mater. 2008, 20, 4749-4755.

[27] Dong, C. PowderX: Windows 95 based program for powder X-ray diffraction data processing. J. Appl. Crystallogr. 1999, 32, 838.

[28] Yu, H. D.; Wang, D. S.; Han, M. Y. Top-down solid-phase fabrication of nanoporous cadmium oxide architectures. $J$. Am. Chem. Soc. 2007, 129, 2333-2337.

[29] Cong, H. P.; Yu, S. H. Shape control of cobalt carbonate particles by a hydrothermal process in a mixed solvent: An efficient precursor to nanoporous cobalt oxide architectures and their sensing property. Cryst. Growth Des. 2009, 9, 210-217. 